\title{
Semiparametric Analysis of Complex Polygenic Gene-Environment Interactions in Case-Control Studies
}

\author{
By Odile Stalder \\ Department of Clinical Research, and Institute of Social and Preventive Medicine, \\ University of Bern, 3012 Bern, Switzerland \\ Odile.Stalder@gmail.com \\ Alex Asher, Liang Liang, Raymond J. Carroll \\ Department of Statistics, Texas A $3 M$ University, College Station, Texas 77843-3143, \\ U.S.A. \\ alexasher@stat.tamu.eduｌiang@stat.tamu.eduｃarroll@stat.tamu.edu \\ YANYUAN MA \\ Department of Statistics, Penn State University, University Park, Pennsylvania 16802, \\ U.S.A. \\ yanyuanma@gmail.com \\ and Nilanjan Chatterjee \\ Department of Biostatistics and Department of Oncology, Johns Hopkins University, \\ Baltimore, Maryland 21205, U.S.A. \\ nchatte2@jhu.edu

\section{SUMmaRY}

Many methods have been recently proposed for efficient analysis of case-control studies of gene-environment interactions using a retrospective likelihood framework that exploits the natural assumption of gene-environment independence in the underlying population. However, for polygenic modeling of gene-environment interactions, a topic of increasing scientific interest, applications of retrospective methods have been limited due to a requirement in the literature for parametric modeling of the distribution of the genetic factors. We propose a general, computationally simple, semiparametric method for analysis of case-control studies that allows exploitation of the assumption of geneenvironment independence without any further parametric modeling assumptions about the marginal distributions of any of the two sets of factors. The method relies on the key observation that an underlying efficient profile likelihood depends on the distribution of genetic factors only through certain expectation terms that can be evaluated empirically. We develop asymptotic inferential theory for the estimator and evaluate numerical performance using simulation studies. An application of the method is presented.

Some key words: Case-control studies; Gene-environment interactions; Genetic epidemiology; Pseudolikelihood; Retrospective studies; Semiparametric methods. 


\section{INTRODUCTION}

Recent genome-wide association studies indicate that complex diseases, such as cancers, diabetes and heart diseases, are in general extremely polygenic (Chatterjee et al.,

40 2016; Fuchsberger et al., 2016). Genetic predisposition to a single disease may involve thousands of genetic variants, each of which may have a very small effect individually, but in combination they can explain substantial variation in risk in the underlying population. As discoveries from genome-wide association studies continue to enhance understanding of complex diseases, in the future, it will be critical to understand how these genetic fac-

45 tors interact with environmental risk factors for both understanding disease mechanisms and developing public health strategies for disease prevention.

Because of its sampling efficiency, the case-control design is widely popular for conducting studies of genetic associations and gene-environment interactions. A variety of analytic methods have been proposed to increase the efficiency of analysis of case-control

5o data for studies of gene-environment interactions by exploiting an assumption of geneenvironment independence in the underlying population. It has been shown that under the assumptions of gene-environment independence and rare disease, the interaction odds-ratio parameters of a logistic regression model can efficiently be estimated based on cases alone (Piegorsch et al., 1994). A general logistic regression model can be fit to case-

55 control data under the gene-environment independence assumption using a log-linear modeling framework (Umbach \& Weinberg, 1997) or a semiparametric retrospective profile likelihood framework (Chatterjee \& Carroll, 2005). More recently, the assumption of gene-environment independence has been exploited to propose a variety of powerful hypothesis testing methods for conducting genome-wide scans of gene-environment interactions (Murcray et al., 2009; Mukherjee \& Chatterjee, 2008; Han et al., 2015; Mukherjee et al., 2012; Gauderman et al., 2013; Hsu et al., 2012).

We consider developing methods for efficient analysis of case-control studies for modeling gene-environment interactions involving multiple genetic variants simultaneously. To develop parsimonious models for joint effects, many studies have recently focused on developing models for gene-environment interactions using underlying polygenic risk scores that could be defined by all known genetic variants associated with the diseases (Meigs et al., 2008; Wacholder et al., 2010; Dudbridge, 2013; Chatterjee et al., 2013, 2016). Further, for obtaining improved biological insights and for enhancing statistical power for detection, it may often be desired to model gene-environment interactions using multiple variants within genomic regions or/and biologic pathways (Chatterjee et al., 2006; Jiao et al., 2013; Lin et al., 2013, 2015). In standard prospective logistic regression analysis, which conditions on both the genetic and environmental risk factor status of the individuals, handling multiple genetic variants is relatively straightforward. In contrast, with retrospective methods, which aim to exploit the assumption of gene75 environment independence, the task becomes complicated because all currently existing methods require parametric modeling of the distribution of the genetic or environmental variables.

We propose computationally simple methodology for fitting general logistic regression models to case-control data under the assumption of gene-environment independence,

so but without requiring any further modeling assumptions about the distributions of the genetic or environmental variables. We extend the Chatterjee-Carroll profile likelihood framework, which originally considered modeling gene-environment interactions using single genetic variants for which genotype status could be specified using parametric 
multinomial models. The new method relies on the observation that the profile likelihood itself can be estimated based on an empirical genotype distribution that is estimable from a case-control sample. We develop the asymptotic theory of the resulting estimator under a semiparametric inferential framework. Simulations and an example illustrate the properties of the new methodology.

\section{Model, Method And Theory}

2.1. Background, model and method

In the following, we use notation similar to that of Chatterjee \& Carroll (2005). We will denote disease status, genetic information and environmental risk factors by $D, G$ and $X$, respectively. Here $G$ may correspond to a complex multivariate genotype associated with multiple genetic variants or a continuous polygenic risk score that is defined a priori based on known associations of the genetic variants with the disease. We assume the risk of the disease given genetic and environmental factors in the underlying population can be specified using a model of the form

$$
\operatorname{pr}(D=1 \mid G, X)=H\left\{\alpha_{0}+m(G, X, \beta)\right\},
$$

where $H(x)=\{1+\exp (-x)\}^{-1}$ is the logistic distribution function and $m(G, X, \beta)$ is a parametrically specified function that defines a model for the joint effect of $G$ and $X$ on the logistic-risk scale. The goal of the gene-environment interaction study is to make inference on the parameters $\beta$ in (1), including interaction parameters.

Let $F(G, X)$ denote the joint distribution of $G$ and $X$ in the underlying population. The key assumption that genetic, $G$, and environmental factors, $X$, are independently distributed in the underlying population can be mathematically stated as

$$
d F(G, X)=d F_{G}(G) \times d F_{X}(X),
$$

where $F_{G}$ and $F_{X}$ denote the underlying marginal distributions of $G$ and $X$, respectively. In the Supplementary Material we discuss how to weaken this assumption by suitable conditioning on additional stratification factors. In contrast to the existing literature, here we assume that the marginal distributions $F_{G}(G)$ and $F_{X}(X)$ are both completely unspecified.

We consider a population-based case-control study, in which $(G, X)$ are sampled independently from those with the disease, called cases, and those without the disease, called controls. Suppose there are $n_{1}$ cases and $n_{0}$ controls. Standard prospective logistic regression analysis, which is equivalent to maximum-likelihood estimation when $F(G, X)$ is allowed to be completely unspecified, yields consistent estimates of $\beta$ (Prentice \& Pyke, 1979).

The retrospective likelihood is the probability of observing the genetic and environmental variables, given the subject's disease status. Under gene-environment independence in the underlying population, the retrospective likelihood is

$$
\operatorname{pr}(G=g, X=x \mid D=d)=\operatorname{pr}(D=d \mid G=g, X=x) \operatorname{pr}(G=g) \operatorname{pr}(X=x) / \operatorname{pr}(D=d) .
$$

Let $f_{G}(\cdot)$ and $f_{X}(\cdot)$ represent the density/mass functions of $G$ and $X$, respectively. The retrospective likelihood is

$$
\frac{f_{G}(g) f_{X}(x) \exp \left[d\left\{\alpha_{0}+m(g, x, \beta)\right\}\right] /\left[1+\exp \left\{\alpha_{0}+m(g, x, \beta)\right\}\right]}{\int f_{G}(u) f_{X}(v) \exp \left[d\left\{\alpha_{0}+m(u, v, \beta)\right\}\right] /\left[1+\exp \left\{\alpha_{0}+m(u, v, \beta)\right\}\right] d u d v} .
$$


Chatterjee \& Carroll (2005) profiled out $f_{X}(\cdot)$ by treating it as discrete on the set of distinct observed values $\left(x_{1}, \ldots, x_{m}\right)$ of $X$ with probabilities $\delta_{i}=\operatorname{pr}\left(X=x_{i}\right)$, and then maximized (2) over $\left(\delta_{1}, \ldots, \delta_{m}\right)$, leading eventually to the semiparametric profile likelihood described as follows. Define $\kappa=\alpha_{0}+\log \left(n_{1} / n_{0}\right)-\log \left(\pi_{1} / \pi_{0}\right)$, where $\pi_{1}=1-\pi_{0}=$ $\operatorname{pr}(D=1)$ is defined as the probability of the disease in the underlying population. Define $\Omega=\left(\kappa, \boldsymbol{\beta}^{\mathrm{T}}\right)^{\mathrm{T}}$. Also define

$$
S(d, g, x, \Omega)=\frac{\exp [d\{\kappa+m(g, x, \beta)\}]}{1+\exp \left\{\kappa+\log \left(\pi_{1} / \pi_{0}\right)-\log \left(n_{1} / n_{0}\right)+m(g, x, \beta)\right\}} .
$$

Then, with this notation, the semiparametric profile likelihood is

$$
L\left(D, G, X, \Omega, f_{G}\right)=f_{G}(G) \frac{S(D, G, X, \Omega)}{\sum_{d=0}^{1} \int f_{G}(v) S(d, v, X, \Omega) d v} .
$$

While the representation in (3) does not involve the unknown density of $X$, it does involve the unknown density of $G$. This is a major reason that the current literature specifies a parametric distribution for $G$. Our task in this paper is to dispense with the need to give a parametric form for the distribution function of $G$, so that analysis can be performed with respect to potentially complex multivariate genotype data for which parametric modeling can be difficult and cumbersome.

Here is our key insight, which we discuss first in the context that $\pi_{1}$ is known or at least can be estimated well. For case-control studies that are conducted within well defined populations, relevant probabilities of the disease can be ascertained based on populationbased disease registries. When case-control studies are conducted by sampling of subjects within a larger cohort study, the probability of the disease in the underlying population can be estimated using the disease incidence rate observed in the cohort.

Our key insight in treating the distribution of $G$ as nonparametric concerns the term in the denominator of (3), defined as

$$
R(x, \Omega)=\sum_{r=0}^{1} \int f_{G}(v) S(r, v, x, \Omega) d v .
$$

This is simply the expectation, in the source population, of $\sum_{r=0}^{1} S(r, G, x, \Omega)$. That is, $R(x, \Omega)=E_{\text {pop }}\left\{\sum_{r=0}^{1} S(r, G, x, \Omega)\right\}$, where the subscript pop emphasizes that the expectation is in the source population, not the case-control study. However, crucially,

$$
R(x, \Omega)=\pi_{1} E\left\{\sum_{r=0}^{1} S(r, G, x, \Omega) \mid D=1\right\}+\pi_{0} E\left\{\sum_{r=0}^{1} S(r, G, x, \Omega) \mid D=0\right\} .
$$

Of course, $R(x, \Omega)$ is unknown, but we estimate it unbiasedly and nonparametrically by

$$
\widehat{R}(x, \Omega)=\sum_{j=1}^{n} \sum_{r=0}^{1} \sum_{d=0}^{1}\left(\pi_{d} / n_{d}\right) I\left(D_{j}=d\right) S\left(r, G_{j}, x, \Omega\right) .
$$

In the Supplementary Material, we show that $\widehat{R}(x, \Omega)$ is an unbiased estimate of $R(x, \Omega)$, that is $n^{1 / 2}$-consistent, and that it is asymptotically normally distributed.

Ignoring the leading term $f_{G}(G)$ in (3), which is not estimated, and taking logarithms, leads us to an estimated loglikelihood in $\Omega$ across the data as

$$
\mathcal{L}(\Omega)=\sum_{i=1}^{n} \log S\left(D_{i}, G_{i}, X_{i}, \Omega\right)-\sum_{i=1}^{n} \log \widehat{R}\left(X_{i}, \Omega\right) .
$$


Define $S_{\Omega}(d, g, x, \Omega)=\partial S(d, g, x, \Omega) / \partial \Omega$ and similarly for $\widehat{R}_{\Omega}(x, \Omega)$. Then the estimated ${ }_{150}$ score function, a type of estimated estimating equation, is

$$
\widehat{\mathcal{S}}_{n}(\Omega)=n^{-1 / 2} \sum_{i=1}^{n}\left\{\frac{S_{\Omega}\left(D_{i}, G_{i}, X_{i}, \Omega\right)}{S\left(D_{i}, G_{i}, X_{i}, \Omega\right)}-\frac{\widehat{R}_{\Omega}\left(X_{i}, \Omega\right)}{\widehat{R}\left(X_{i}, \Omega\right)}\right\} .
$$

Define

$$
\mathcal{S}_{n}(\Omega)=n^{-1 / 2} \sum_{i=1}^{n}\left\{\frac{S_{\Omega}\left(D_{i}, G_{i}, X_{i}, \Omega\right)}{S\left(D_{i}, G_{i}, X_{i}, \Omega\right)}-\frac{R_{\Omega}\left(X_{i}, \Omega\right)}{R\left(X_{i}, \Omega\right)}\right\}
$$

which is the profile loglikelihood score function when the distribution of $G$ is known. Since the profile loglikelihood score of Chatterjee \& Carroll (2005) would have mean zero if the distribution of $G$ were known, it follows that

$$
E\left\{\mathcal{S}_{n}(\Omega)\right\}=0
$$

where the expectation in (8) is taken in the case-control study, not in the source population. Thus, since $\widehat{R}(x, \Omega)$ and $\widehat{R}_{\Omega}(x, \Omega)$ converge in probability to $R(x, \Omega)$ and $R_{\Omega}(x, \Omega)$, respectively, a consistent estimate of $\Omega$ can be obtained by solving $\widehat{\mathcal{S}}_{n}(\Omega)=0$. This estimate $\widehat{\Omega}$, which maximizes the semiparametric pseudolikelihood (6), will be referred to as the semiparametric pseudolikelihood estimator.

\subsection{Rare diseases when $\pi_{1}$ is unknown}

When the probability of disease in the source population is unknown, one can invoke a rare disease assumption which is often reasonable for case-control studies (Piegorsch et al., 1994; Modan et al., 2001; Epstein \& Satten, 2003; Lin \& Zeng, 2006; Kwee et al., 2007; Zhao et al., 2003). If we assume that $\pi_{1} \approx 0$, then $S(d, g, x, \Omega) \approx$ $\exp [d\{\kappa+m(g, x, \beta\}]$, and the expectation involved in calculation of $R(X, \Omega)$ can be evaluated based on the sample of controls, with $D=0$, only. In this case, the estimates of $\Omega$ converge not to $\Omega$ itself, but instead to $\Omega_{*}$, the solution to (8) with $\pi_{1}=0$. Typically, except when the sample size is very large and hence standard errors are unusually small, the small possible bias of the rare disease approximation is of little consequence and coverage probabilities of confidence intervals remain near nominal, see $\S 3$ for examples. The asymptotic theory of $\S 2 \cdot 3$ below is then unchanged.

In the Supplementary Material, we show that the score and the Hessian take on simple forms in this case, and that the Hessian is negative semidefinite. Computation is thus very efficient.

\subsection{Asymptotic theory}

To state the asymptotic results, we first make the definitions

$$
\begin{aligned}
& \Gamma_{1}=\sum_{d=0}^{1}\left(n_{d} / n\right) E\left\{\frac{\partial S_{\Omega}(D, G, X, \Omega) / S(D, G, X, \Omega)}{\partial \Omega^{\mathrm{T}}} \mid D=d\right\} ; \\
& \Gamma_{2}=\sum_{d=0}^{1}\left(n_{d} / n\right) E\left\{\frac{\partial R_{\Omega}(X, \Omega) / R(X, \Omega)}{\partial \Omega^{\mathrm{T}}} \mid D=d\right\} .
\end{aligned}
$$

In addition, define $c_{d}=n_{d} / n, \quad Z_{i}=\left(D_{i}, G_{i}, X_{i}\right), \quad P_{1}\left(X_{i}, \Omega\right)=1 / R\left(X_{i}, \Omega\right) \quad$ and $P_{2}\left(X_{i}, \Omega\right)=R_{\Omega}\left(X_{i}, \Omega\right) / R^{2}\left(X_{i}, \Omega\right)$. 
We use the notational convention that for arbitrary functions $(P, T), T_{E}(r, d, x)=$ $E\{T(r, G, x) \mid D=d\}$. Also, we use the convention that

$$
\begin{aligned}
& E\left[P(X)\left\{T\left(r, g_{i}, X\right)-T_{E}(r, d, X)\right\} \mid D=t\right] \\
& \quad=E\left[P(X)\left\{T(r, g, X)-T_{E}(r, d, X)\right\} \mid D=t\right]_{g=G_{i}}
\end{aligned}
$$

Define

$$
\begin{aligned}
\zeta\left(Z_{i}, \Omega\right)= & \frac{S_{\Omega}\left(Z_{i}, \Omega\right)}{S\left(Z_{i}, \Omega\right)}-\frac{R_{\Omega}\left(X_{i}, \Omega\right)}{R\left(X_{i}, \Omega\right)} \\
& -\sum_{d=0}^{1} \sum_{r=0}^{1} \frac{c_{d} \pi_{d_{i}}}{c_{d_{i}}} E\left[\left\{P_{1}(X, \Omega) S_{\Omega}\left(r, g_{i}, X\right)-P_{2}(X, \Omega) S\left(r, g_{i}, X\right)\right\} \mid D=d\right] .
\end{aligned}
$$

Finally, define $\zeta_{*}\left(Z_{i}, \Omega\right)=\zeta\left(Z_{i}, \Omega\right)-E\left\{\zeta(Z, \Omega) \mid D=D_{i}\right\}$.

Theorem 1. Suppose $n_{d} / n \rightarrow c_{d}$, where $0<c_{d}<\infty$, and that $\pi_{1}$ is known. Then

$$
n^{1 / 2}(\widehat{\Omega}-\Omega)=-\left(\Gamma_{1}-\Gamma_{2}\right)^{-1} n^{-1 / 2} \sum_{i=1}^{n} \zeta_{*}\left(Z_{i}, \Omega\right)+o_{p}(1) .
$$

Thus, since the $Z_{i}$ are independent and $E\left\{\zeta_{*}(Z, \Omega) \mid D_{i}\right\}=0$,

$$
\begin{aligned}
n^{1 / 2}(\widehat{\Omega}-\Omega) & \rightarrow N\left[0,\left(\Gamma_{1}-\Gamma_{2}\right)^{-1} \Sigma\left\{\left(\Gamma_{1}-\Gamma_{2}\right)^{-1}\right\}^{\mathrm{T}}\right] \\
\Sigma & =\sum_{d=0}^{1}\left(n_{d} / n\right) \operatorname{cov}\left\{\zeta_{*}(D, X, G, \Omega) \mid D=d\right\} \\
& =\sum_{d=0}^{1}\left(n_{d} / n\right) \operatorname{cov}\{\zeta(D, X, G, \Omega) \mid D=d\} .
\end{aligned}
$$

In $\S 2 \cdot 2$, when $\pi_{1}$ is unknown and the disease is relatively rare, the same result holds by setting $\pi_{1}=0$.

\section{Simulations}

\section{$3 \cdot 1$. Overview}

190

In our simulations, $m(G, X, \beta)=G^{\mathrm{T}} \beta_{G}+X \beta_{X}+(G X)^{\mathrm{T}} \beta_{G X}$ and the value of $X$ is binary with population frequency 0.5 . There are either three or five correlated single nucleotide polymorphisms within a region: we report the latter case, but the results for the former are similar. Each single nucleotide polymorphism takes on the values 0,1 or 2 following a trinomial distribution that follows Hardy-Weinberg equilibrium, i.e., the $j$ th component of $G$ equals $0,1,2$ with probabilities $\left\{\left(1-p_{j}\right)^{2}, 2 p_{j}\left(1-p_{j}\right), p_{j}^{2}\right\}$. The values of the $p_{j}$ are described below.

To generate correlation among the single nucleotide polymorphisms, we first generated a 3 or 5 -variate multivariate normal variate, each with mean 0 and standard deviation 1 , and a correlation matrix with correlation between the $j$ th and $k$ th component $=\rho^{|j-k|}$, where $\rho=0.7$. After generating these random variables, we trichotomized them with appropriate thresholds so that frequency of 0,1 and 2 matched those specified by the allele frequency $p_{j}$ and Hardy-Weinberg equilibrium. 
Table 1: Results of 1000 simulations as described in $\S 3$, with mean bias, coverage probabilities of a $95 \%$ nominal confidence interval, and mean squared error efficiency of our semiparametric pseudolikelihood estimator compared to ordinary logistic regression. The simulations were performed with 1000 cases and 1000 controls.

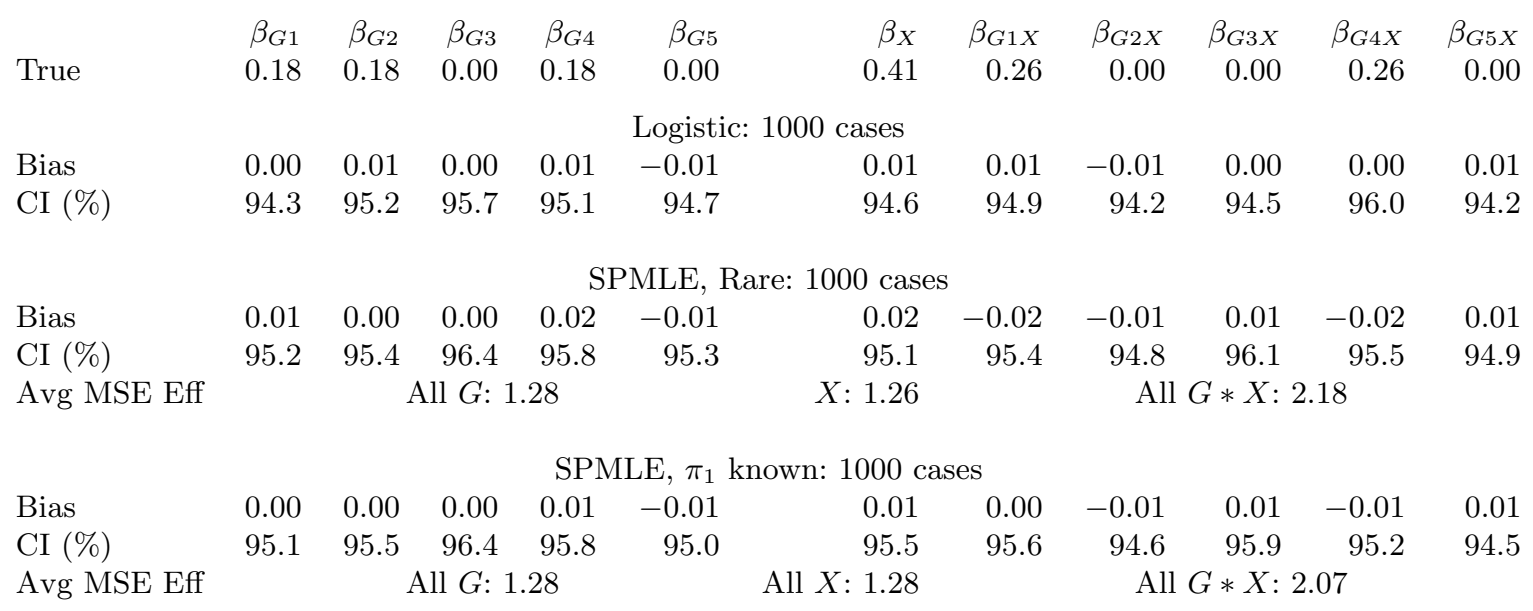

Logistic is ordinary logistic regression; $S P M L E$, Rare is our estimator using the rare disease approximation with unknown $\pi_{1}(\S 2 \cdot 2)$; SPMLE, $\pi_{1}$ known is our estimator when $\pi_{1}$ is known in the source population $(\S 2 \cdot 1) ; C I(\%)$ is the coverage in percent of a nominal $95 \%$ confidence interval (calculated using the asymptotic standard error); Avg MSE Eff is the mean squared error efficiency of our method compared to logistic regression averaged over $G$ (All $G$ ), over $X($ All $X)$ and over the $G * X($ All $G * X)$ interactions.

In both simulations, the logistic intercept $\alpha_{0}$ was chosen so that the population disease rate $\pi_{1}=0.03$. However additional simulations with $\pi_{1}=0.01$ yielded very similar results with regards to coverage, efficiency gains, and unbiasedness. See also $\S 3 \cdot 3$ for a discussion of additional simulations, and the Supplementary Material. In the simulation reported here, $\left(p_{1}, p_{2}, p_{3}, p_{4}, p_{5}\right)=(0.1,0.3,0.3,0.3,0.1), \beta_{X}=\log (1.5), \beta_{G}=$ $\{\log (1.2), \log (1.2), 0.0, \log (1.2), 0.0\}$, and $\beta_{G X}=\{\log (1.3), 0.0,0.0, \log (1.3), 0.0\}$. Here the value of $\alpha_{0}=-4.14$.

\subsection{Results}

The standard error estimators used in our simulation were based on the asymptotic theory described in Theorem 1: we also used the bootstrap, with very similar results. The appropriate bootstrap in a case-control study is to resample the cases and controls separately, thus maintaining the sample sizes for each.

The simulation results are presented in Table 1. Our semiparametric pseudolikelihood estimator has little bias and coverage percentages near the nominal level. Both with a rare disease approximation and with $\pi_{1}$ known, our semiparametric pseudolikelihood estimator achieves approximately a $25 \%$ increase in mean squared error efficiency over ordinary logistic regression for the main effects in both $G$ and $X$.

Strikingly, the mean squared error efficiency of our semiparametric pseudolikelihood estimators compared to ordinary logistic regression is approximately 2.00 for all the interaction terms, thus demonstrating that our methods, which do not model the distribution of either $G$ or $X$, achieve numerically significant increases in efficiency. 


\subsection{Additional simulations}

The Supplementary Material presents a series of additional simulations. These include the results of a simulation to evaluate the robustness of our method to misspecification of the population disease rate, where we found a surprising robustness to disease rate misspecification. Additionally, there are simulations to examine the robustness of our method to violations of the gene-environment independence assumption. Those simulation studies show that there will be bias in the estimate of gene-environment interaction parameters for the specific single nucleotide polymorphisms under violation of gene-environment independence, but average mean square error for parameter estimates across all the different single nucleotide polymorphisms could be still substantially lower than that obtained from prospective logistic regression analysis. We also show there how to remove this bias when $\mathrm{G}$ and $\mathrm{E}$ are independent conditional on a discrete stratification variable. Mukherjee \& Chatterjee (2008) and Chen et al. (2009) show how to use empirical-Bayes methods to provide additional robustness to violations of the geneenvironment independence assumption.

\section{Data Analysis}

In this section, we apply our methodology to a case-control study for breast cancer arising from a large prospective cohort at the National Cancer Institute: the Prostate, Lung, Colorectal and Ovarian cancer screening trial (Canzian et al., 2010). The design of this study is described in detail by Prorok et al. (2000) and Hayes et al. (2000). The cohort data consisted of 622,449 women, of whom $3.56 \%$ developed breast cancer (Pfeiffer et al., 2013). The case-control study analyzed here consists of 753 controls and 658 cases. Although $\pi_{1}$ is known in this population, we analyze the data both with $\pi_{1}$ known and with $\pi_{1}$ unknown but with a rare disease approximation.

We had data available on genotypes for 21 single nucleotide polymorphisms that have been previously associated with breast cancer based on large genome-wide association studies. The polygenic risk score was defined by a weighted combination of the genotypes, with the weights defined by log-odds-ratio coefficients reported in prior studies. We examined the interaction of the polygenic risk score with age at menarche $(X)$, a known risk factor for breast cancer, defined as the binary indicator of whether the age at menarche exceeds 13 or not. We also adjust the model for age as a continuous variable, denoted here as $Z$, so that the model fitted is

$$
\operatorname{pr}(D=1)=H\left(\beta_{0}+\beta_{G} G+\beta_{X} X+\beta_{G X} G X+\beta_{Z} Z\right) .
$$

Results in which age was categorized as $<35,35-40,40-45, \ldots,>75$ were similar.

We also performed analyses to check the gene-environment independence assumption. Since $X$ is binary, we ran a t-test of the polygenic risk score against the levels of $X$, of course among the controls only. The p-value was 0.91 , indicating almost no genetic effect. We also ran chisquared tests for the 21 individual genes, finding no significant association after controlling the false discovery rate: the minimum q-value was 0.09 . We also checked for correlation, known as linkage disequilibrium, between the 21 loci used to create the polygenic risk score and 32 loci that are known to influence age at menarche (Elks et al., 2010). The data available to us do not have the necessary information to analyze linkage disequilibrium between the two sets of loci. 
Table 2: Results of the analysis of the Prostate, Lung, Colorectal and Ovarian cancer screening trial data

$\begin{array}{lllll} & \beta_{Z} & \beta_{G} & \beta_{X} & \beta_{G X} \\ \text { Logistic } & & & & \\ \quad \text { Estimate } & 0.018 & 0.297 & -0.165 & 0.124 \\ \quad \text { std err } & 0.054 & 0.064 & 0.132 & 0.068 \\ \quad \text { p-value } & 7.45 \times 10^{-1} & 3.19 \times 10^{-6} & 2.10 \times 10^{-1} & 6.87 \times 10^{-2} \\ & & & & \\ \text { SPMLE, Rare } & & & & \\ \quad \text { Estimate } & 0.024 & 0.321 & -0.175 & 0.138 \\ \quad \text { std err (asymptotic) } & 0.054 & 0.067 & 0.134 & 0.055 \\ \text { p-value (asymptotic) } & 6.60 \times 10^{-1} & 1.62 \times 10^{-6} & 1.91 \times 10^{-1} & 1.16 \times 10^{-2} \\ & & & & \\ \text { SPMLE, } \pi_{1} \text { known } & & & & 0.141 \\ \quad \text { Estimate } & 0.022 & 0.313 & -0.174 & 0.055 \\ \text { std err (asymptotic) } & 0.054 & 0.065 & 0.133 & 1.13 \times 10^{-2} \\ \text { p-value (asymptotic) } & 6.78 \times 10^{-1} & 1.64 \times 10^{-6} & 1.93 \times 10^{-1} & \end{array}$

Logistic is ordinary logistic regression; SPMLE, Rare is our method using the rare disease approximation with unknown $\pi_{1}$; SPMLE, $\pi_{1}$ known is our method when the disease rate is known in the source population $\left(\pi_{1}=3.56 \%\right)$; std err is the asymptotic standard error estimate; $\beta_{Z}$ is the main effect for age; $\beta_{G}$ and $\beta_{X}$ are the main effects for the polygenic risk score $(G)$ and the environmental variable $X$ (age at menarche $>$ 13), respectively; $\beta_{G X}$ is the gene-environment interaction.

Using phased haplotypes from subjects of European descent from 1000 Genomes (The 1000 Genomes Project Consortium, 2015) and HapMap (Gibbs et al., 2003), no evidence of linkage disequilibrium was found: the maximum $R^{2}$ was 0.1 and the minimum q-value was 0.85 . Finally, a 2014 study examined the relationship between age at menarche and 10 of the 21 SNPs used to create our polygenic risk score, none of which were found to influence age at menarche (Andersen et al., 2014).

Table 2 presents the results for the cases that $\pi_{1}$ is unknown and known, respectively: as remarked upon previously, the results are very similar. To provide a basis for comparison because of the very different scales of the variables, the variable age at baseline was standardized to have mean zero and standard deviation one. In addition, we standardized some of the coefficient estimates so that $\beta_{G}$ was multiplied by the standard deviation of the polygenic risk score, and $\beta_{G X}$ was multiplied by the standard deviation of $X$ times the polygenic risk score.

As expected from the known association of the single nucleotide polymorphisms with risk of breast cancer, the polygenic risk score was strongly associated with breast cancer status of the women in the study. Standard logistic regression analysis reveals some evidence for interaction of the polygenic risk score with age-at-menarche, but the result was not statistically significant at the 0.05 level. When the analysis was done under the gene-environment independence assumption, the evidence of interaction appeared to be stronger.

The coefficient estimate for the interaction term is slightly larger for our semiparametric methods than that for logistic regression. Also, the asymptotic standard error estimate of logistic regression is approximately $23 \%$ larger than our methods, indicating a variance increase of $\approx 50 \%$. Although not listed here, the balanced bootstrap mentioned in $\S 3 \cdot 2$ has very similar standard error estimates. In that bootstrap, $33 \%$ of the 
time, the logistic interaction estimate was actually greater than that of the disease rate known estimate.

\section{Discussion AND Extensions}

We have proposed a general method for using retrospective likelihoods for studying

\section{Science Foundation and the National} Science Foundation and the National Institute of Neurological Disorders and Stroke. Asher, Liang and Carroll's research was supported by the National Cancer Institute.

\section{SUPPLEMENTARY MATERIAL}

Supplementary Material available at Biometrika online contains proofs, skewness and kurtosis and q-q plots for the simulation in Table 1, how to modify our methods to account for strata, additional simulations and software written in R. The data used in $\S 4$ are available from the National Cancer Institute via a data transfer agreement. 


\section{REFERENCES}

Andersen, S. W., Trentham-Dietz, A., Gangnon, R. E., Hampton, J. M., Skinner, H. G., Engelman, C. D., Klein, B. E., Titus, L. J., Egan, K. M. \& Newcomb, P. A. (2014). Breast cancer susceptibility loci in association with age at menarche, age at natural menopause and the reproductive lifespan. Cancer Epidemiol. 38, 62-65.

Canzian, F., Cox, D. G., Setiawan, V. W., Stram, D. O., Ziegler, R. G., Dossus, L., Beckmann, L., Blanché, H., Barricarte, A., Berg, C. D. et al. (2010). Comprehensive analysis of common genetic variation in 61 genes related to steroid hormone and insulin-like growth factor-i metabolism and breast cancer risk in the NCI breast and prostate cancer cohort consortium. Hum. Mol. Genet. 19, 3873-3884.

Chatterjee, N. \& Carroll, R. J. (2005). Semiparametric maximum likelihood estimation in casecontrol studies of gene-environment interactions. Biometrika 92, 399-418.

Chatterjee, N., Kalaylioglu, Z., Moslehi, R., Peters, U. \& Wacholder, S. (2006). Powerful multilocus tests of genetic association in the presence of gene-gene and gene-environment interactions. Am. J. Hum. Genet. 79, 1002-1016.

Chatterjee, N., Shi, J. \& García-Closas, M. (2016). Developing and evaluating polygenic risk prediction models for stratified disease prevention. Nature Rev. Genet. 17, 392-406.

Chatterjee, N., Wheeler, B., Sampson, J., Hartge, P., Chanock, S. J. \& Park, J.-H. (2013). Projecting the performance of risk prediction based on polygenic analyses of genome-wide association studies. Nature Genet. 45, 400-405.

Chen, Y. H., Chatterjee, N. \& Carroll, R. J. (2008). Retrospective analysis of haplotype-based case-control studies under a flexible model for gene-environment association. Biostatistics 9, 81-99.

Chen, Y. H., Chatterjee, N. \& Carroll, R. J. (2009). Shrinkage estimators for robust and efficient inference in haplotype-based case-control studies. J. Am. Statist. Assoc. 104, 220-233.

Dudbridge, F. (2013). Power and predictive accuracy of polygenic risk scores. PLoS Genet. 9, e1003348.

Elks, C. E., Perry, J. R. B., Sulem, P., Chasman, D. I., Franceschini, N., He, C., Lunetta, K. L., Visser, J. A., Byrne, E. M., Cousminer, D. L. et al. (2010). Thirty new loci for age at menarche identified by a meta-analysis of genome-wide association studies. Nature Genet. 42, 1077-1085.

Epstein, M. P. \& Satten, G. A. (2003). Inference on haplotype effects in case-control studies using unphased genotype data. Am. J. Hum. Genet. 73, 1316-1329.

Fuchsberger, C., Flannick, J., Teslovich, T. M. et al. (2016). The genetic architecture of type 2 diabetes. Nature (doi: 10.1038/nature18642).

Gauderman, W. J., Zhang, P., Morrison, J. L. \& Lewinger, J. P. (2013). Finding novel genes by testing G×E interactions in a Genome-Wide Association Study. Genet. Epidemiol. 37, 603-613.

Gibbs, R. A., Belmont, J. W., Hardenbol, P., Willis, T. D., Yu, F., Yang, H., Ch'ang, L.-Y., Huang, W., Liu, B., Shen, Y. et al. (2003). The International HapMap Project. Nature 426, 789-796.

Han, S. S., Rosenberg, P. S., Ghosh, A., Landi, M. T., Caporaso, N. E. \& Chatterjee, N. (2015). An exposure-weighted score test for genetic associations integrating environmental risk factors. Biometrics 71, 596-605.

Hayes, R. B., Reding, D., Kopp, W., Subar, A. F., Bhat, N., Rothman, N., Caporaso, N., Ziegler, R. G., Johnson, C. C., Weissfeld, J. L. et al. (2000). Etiologic and early marker studies in the Prostate, Lung, Colorectal and Ovarian (PLCO) cancer screening trial. Control. Clin. Trials 21, 349S-355S.

Hsu, L., Jiao, S., Dai, J. Y., Hutter, C., Peters, U. \& Kooperberg, C. (2012). Powerful cocktail methods for detecting genome-wide gene-environment interaction. Genet. Epidemiol. 36, 183-194.

Jiao, S., Hsu, L., Bézieau, S., Brenner, H., Chan, A. T., Chang-Claude, J., Le Marchand, L., Lemire, M., Newcomb, P. A., Slattery, M. L. et al. (2013). SBERIA: Set-based gene-environment interaction test for rare and common variants in complex diseases. Genet. Epidemiol. 37, 452-464.

Kwee, L. C., Epstein, M. P., Manatunga, A. K., Duncan, R., Allen, A. S. \& Satten, G. A. (2007). Simple methods for assessing haplotype-environment interactions in case-only and case-control studies. Genet. Epidemiol. 31, 75-90.

Lin, D. Y. \& ZENG, D. (2006). Likelihood-based inference on haplotype effects in genetic association studies. J. Am. Statist. Assoc. 101, 89-104.

Lin, D. Y. \& ZENG, D. (2009). Proper analysis of secondary phenotype data in case-control association studies. Genet. Epidemiol. 33, 256-265.

Lin, X., Lee, S., Christiani, D. C. \& Lin, X. (2013). Test for interactions between a genetic marker set and environment in generalized linear models. Biostatistics 14, 667-681.

Lin, X., Lee, S., Wu, M. C., Wang, C., Chen, H., Li, Z. \& Lin, X. (2015). Test for rare variants by environment interactions in sequencing association studies. Biometrics 72, 156-164.

MA, Y. (2010). A semiparametric efficient estimator in case-control studies. Bernoulli 16, 585-603. 
Meigs, J. B., Shrader, P., Sullivan, L. M., McAteer, J. B., Fox, C. S., Dupuis, J., Manning, A. K., Florez, J. C., Wilson, P. W., D'Agostino SR, R. B. et al. (2008). Genotype score in addition to common risk factors for prediction of type 2 diabetes. N. Engl. J. Med. 359, 2208-2219.

Modan, B., Hartge, P., Hirsh-Yechezkel, G., Chetrit, A., Lubin, F., Beller, U., Ben-Baruch, G., Fishman, A., Menczer, J., Struewing, J. P. et al. (2001). Parity, oral contraceptives, and the risk of ovarian cancer among carriers and noncarriers of a BRCA1 or BRCA2 mutation. N. Engl. J. Med. 345, 235-240.

Mukherjee, B., Ahn, J., Gruber, S. B. \& Chatterjee, N. (2012). Testing gene-environment interaction in large-scale case-control association studies: possible choices and comparisons. Am. J. Epidemiol. 175, 177-190.

400 Mukherjee, B. \& Chatterjee, N. (2008). Exploiting gene-environment independence for analysis of case-control studies: An empirical bayes-type shrinkage estimator to trade-off between bias and efficiency. Biometrics 64, 685-694.

Murcray, C. E., Lewinger, J. P. \& Gauderman, W. J. (2009). Gene-environment interaction in genome-wide association studies. Am. J. Epidemiol. 169, 219-226.

NEWEY, W. K. (1994). The asymptotic variance of semiparametric estimators. Econometrica 62, 13491382.

Pfeiffer, R. M., Park, Y., Kreimer, A. R., Lacey Jr, J. V., Pee, D., Greenlee, R. T., Buys, S. S., Hollenbeck, A., Rosner, B., Gail, M. H. et al. (2013). Risk prediction for breast, endometrial, and ovarian cancer in white women aged 50 y or older: derivation and validation from population-based cohort studies. PLoS Med. 10, e1001492.

Piegorsch, W. W., Weinberg, C. R. \& Taylor, J. A. (1994). Non-hierarchical logistic models and case-only designs for assessing susceptibility in population based case-control studies. Statist. Med. 13, 153-162.

Prentice, R. L. \& Pyke, R. (1979). Logistic disease incidence models and case-control studies. Biometrika 66, 403-411.

Prorok, P. C., Andriole, G. L., Bresalier, R. S., Buys, S. S., Chia, D., Crawford, E. D., Fogel, R., Gelmann, E. P., Gilbert, F., Hasson, M. A. et al. (2000). Design of the prostate, lung, colorectal and ovarian (PLCO) cancer screening trial. Control. Clin. Trials 21, 273S-309S.

The 1000 Genomes Project Consortium (2015). A global reference for human genetic variation. Nature 526, 68-74.

Umbach, D. M. \& Weinberg, C. R. (1997). Designing and analysing case-control studies to exploit independence of genotype and exposure. Statist. Med. 16, 1731-1743.

Wacholder, S., Hartge, P., Prentice, R., Garcia-Closas, M., Feigelson, H. S., Diver, W. R., Thun, M. J., Cox, D. G., Hankinson, S. E., Kraft, P. et al. (2010). Performance of common genetic variants in breast-cancer risk models. N. Engl. J. Med. 362, 986-993.

ZhaO, L. P., Li, S. S. \& Khalid, N. (2003). A method for the assessment of disease associations with single-nucleotide polymorphism haplotypes and environmental variables in case-control studies. $\mathrm{Am}$. J. Hum. Genet. 72, 1231-1250. 